\title{
Alternative swabs and storage for SARS-CoV-2 detection in a hospital environment
}

Jeremiah J. Minich

Farhana Ali

Clarisse Marotz

Pedro Belda-Ferre

Leslie Chiang

Justin P. Shaffer

Carolina S. Carpenter

Daniel McDonald

Jack Gilbert

Sarah M. Allard

Eric E Allen

Rob Knight

Daniel A. Sweeney

Austin D. Swafford

\section{Video Byte}

Keywords: COVID-19, SARS-CoV-2, RT-qPCR, swab, global health, hospital, diagnosis, diagnostic test, Microbiome

Posted Date: February 25th, 2021

DOI: https://doi.org/10.21203/rs.3.rs-276068/v1

License: (9) This work is licensed under a Creative Commons Attribution 4.0 International License. Read Full License 


\section{Abstract}

Since its appearance in late 2019, COVID-19 has caused well over one million deaths worldwide. Largescale testing and contact tracing remain critical for controlling viral spread. Complying with the US CDC and WHO protocols for sample collection requires a ready supply of inexpensive swabs and collection reagents. Unfortunately, CDC-approved clinical-grade sampling supplies are expensive, and additionally, current methods prevent further analysis of the microbiome due to the presence of antibiotics in viral transport media. Researchers sought out new testing supplies in a recent study comparing five consumergrade swabs and one clinical-grade swab. They found that using $95 \%$ ethanol instead of viral transfer media reduced RNase activity, preserving samples for microbiome analysis, and extracting directly from the swab head instead of the surrounding liquid resulted in 2-4x higher RNA recovery than the clinical standard. The limit of detection was similar for samples collected with consumer-grade or research-grade plastic swabs compared to the CDC-approved synthetic swab, and microbiome analysis demonstrated that microbial communities were not impacted by swab type. These results suggest that improved SARSCoV-2 detection may be possible using a less expensive technique that also preserves material for microbiome analysis. 